ANNALES

POLONICI MATHEMATICI

$93.1(2008)$

\title{
On the zero set of the Kobayashi-Royden pseudometric of the spectral unit ball
}

\author{
by Nikolai Nikolov (Sofia) and Pascal J. Thomas (Toulouse)
}

\begin{abstract}
Given $A \in \Omega_{n}$, the $n^{2}$-dimensional spectral unit ball, we show that if $B$ is an $n \times n$ complex matrix, then $B$ is a "generalized" tangent vector at $A$ to an entire curve in $\Omega_{n}$ if and only if $B$ is in the tangent cone $C_{A}$ to the isospectral variety at $A$. In the case of $\Omega_{3}$, the zero set of the Kobayashi-Royden pseudometric is completely described.
\end{abstract}

1. Introduction and results. Let $\mathcal{M}_{n}$ be the set of all $n \times n$ complex matrices. For $A \in \mathcal{M}_{n}$ denote by $\operatorname{sp}(A)$ and $r(A)=\max _{\lambda \in \operatorname{sp}(A)}|\lambda|$ the spectrum and the spectral radius of $A$, respectively. The spectral ball $\Omega_{n}$ is the set

$$
\Omega_{n}:=\left\{A \in \mathcal{M}_{n}: r(A)<1\right\} .
$$

The spectral Nevanlinna-Pick problem is the following: given $N$ points $a_{1}, \ldots, a_{N}$ in the unit disk $\mathbb{D} \subset \mathbb{C}$ and $N$ matrices $A_{1}, \ldots, A_{N} \in \Omega_{n}$ decide whether there is a mapping $\varphi \in \mathcal{O}\left(\mathbb{D}, \Omega_{n}\right)$ such that $\varphi\left(a_{j}\right)=A_{j}, 1 \leq j \leq N$ (cf. $[1,2,4,7,8]$ and the references there).

The study of the Nevanlinna-Pick problem in the case $N=2$ reduces to the computation of the Lempert function, defined as follows for a domain $D \subset \mathbb{C}^{m}:$

$$
l_{D}(z, w):=\inf \{|\alpha|: \exists \varphi \in \mathcal{O}(\mathbb{D}, D): \varphi(0)=z, \varphi(\alpha)=w\}, \quad z, w \in D .
$$

The infinitesimal version of the above is the Carathéodory-Fejér problem of order 1: given matrices $A_{0}, A_{1} \in \mathcal{M}_{n}$, decide whether there is a mapping $\varphi \in \mathcal{O}\left(\mathbb{D}, \Omega_{n}\right)$ such that $A_{0}=\varphi(0), A_{1}=\varphi^{\prime}(0)$. This problem has been studied in [10].

2000 Mathematics Subject Classification: Primary 32F45; Secondary 32A07.

Key words and phrases: spectral Nevanlinna-Pick problem, spectral CarathéodoryFejér problem, spectral ball, symmetrized polydisc, Lempert function, Kobayashi-Royden pseudometric.

The initial version of this paper was written during the stay of the first named author at the Paul Sabatier University, Toulouse in May-June, 2007. 
Its study reduces to the computation of the Kobayashi-Royden pseudometric, defined as follows for a domain $D \subset \mathbb{C}^{m}$ :

$$
\begin{aligned}
& k_{D}(z ; X):=\inf \{|\alpha|: \exists \varphi \in \mathcal{O}(\mathbb{D}, D): \\
& \left.\quad \varphi(0)=z, \alpha \varphi^{\prime}(0)=X\right\}, \quad z \in D, X \in \mathbb{C}^{m} .
\end{aligned}
$$

To each matrix $A$ we associate its characteristic polynomial

$$
P_{A}(t):=\operatorname{det}(t I-A)=t^{n}+\sum_{j=1}^{n}(-1)^{j} \sigma_{j}(A) t^{n-j},
$$

where $I \in \mathcal{M}_{n}$ is the unit matrix,

$$
\sigma_{j}(A):=\sigma_{j}\left(\lambda_{1}, \ldots, \lambda_{n}\right):=\sum_{1 \leq k_{1}<\cdots<k_{j} \leq n} \lambda_{k_{1}} \ldots \lambda_{k_{j}}
$$

and $\lambda_{1}, \ldots, \lambda_{n}$ are the eigenvalues of $A$.

Put $\sigma:=\left(\sigma_{1}, \ldots, \sigma_{n}\right): \mathcal{M}_{n} \rightarrow \mathbb{C}^{n}$. The set

$$
\mathbb{G}_{n}:=\left\{\sigma(A): A \in \Omega_{n}\right\}
$$

is a taut (even hyperconvex) domain called the symmetrized $n$-disk (cf. [3, $8,11]$ and references there). Explicit formulas for $l_{\mathbb{G}_{2}}$ and $k_{\mathbb{G}_{2}}$ can be found in [2] (see also [11]) and [10], respectively.

Recall now that a matrix $A \in \mathcal{M}_{n}$ is called nonderogatory if all the blocks in the Jordan form of $A$ have distinct eigenvalues. Many properties equivalent to this definition may be found in [13, Proposition 3]. We point out one of them: $A$ is nonderogatory if and only if $\operatorname{rank}\left(\sigma_{*, A}\right)=n$, where $\sigma_{*, A}$ stands for the differential of $\sigma$ at the point $A$.

Denote by $\mathcal{C}_{n}$ the open and dense set of all nonderogatory matrices in $\Omega_{n}$.

If $A_{1}, \ldots, A_{N} \in \mathcal{C}_{n}$, then any mapping $\varphi \in \mathcal{O}\left(\mathbb{D}, \mathbb{G}_{n}\right)$ with $\varphi\left(\alpha_{j}\right)=\sigma\left(A_{j}\right)$ can be lifted to a mapping $\widetilde{\varphi} \in \mathcal{O}\left(\mathbb{D}, \Omega_{n}\right)$ with $\widetilde{\varphi}\left(\alpha_{j}\right)=A_{j}, 1 \leq j \leq N$ (see [1]). This means that in a generic case the spectral NevanlinnaPick problem for $\Omega_{n}$ (with dimension $n^{2}$ ) can be reduced to the standard Nevanlinna-Pick problem for $\mathbb{G}_{n}$ (with dimension $n$ ).

By the contractibility of the Lempert function, we have

$$
l_{\Omega_{n}}(A, B) \geq l_{\mathbb{G}_{n}}(\sigma(A), \sigma(B)), \quad A, B \in \Omega_{n},
$$

and the lifting above implies that equality holds when $A, B \in \mathcal{C}_{n}$.

From this and the fact that $\mathbb{G}_{n}$ is a bounded domain, $l_{\Omega_{n}}(A, B)>0$ if $\operatorname{sp}(A) \neq \operatorname{sp}(B)$. On the other hand, if $\operatorname{sp}(A)=\operatorname{sp}(B)$, then there is an entire mapping $\varphi: \mathbb{C} \rightarrow \Omega_{n}$ with $\varphi(0)=A$ and $\varphi(1)=B$ (see [9]); note that $\operatorname{sp}(\varphi(\zeta))=\operatorname{sp}(A)$ for all $\zeta \in \mathbb{C}$, since by Liouville's theorem, whenever $\varphi(\mathbb{C}) \subset \Omega$, then $\sigma \circ \varphi$ is constant. This situation is similar to that of Brody's theorem for compact manifolds [5]: failure of hyperbolicity (that is, vanishing 
of the pseudodistance) can be explained by the presence of a (nonconstant) entire curve in the manifold.

Restricting again to nonderogatory matrices, a similar lifting [10] implies in the Carathéodory-Fejér case

$$
k_{\Omega_{n}}(A ; B)=k_{\mathbb{G}_{n}}\left(A, \sigma_{*, A}(B)\right), \quad A \in \mathcal{C}_{n}, B \in \mathcal{M}_{n} ;
$$

in particular, $k_{\Omega_{n}}(A ; B)=0$ if and only if $\sigma_{*, A}(B)=0$. On the other hand, if $\sigma_{*, A}(B)=0$, then there is an entire mapping $\varphi: \mathbb{C} \rightarrow \Omega_{n}$ with $\varphi(0)=A$ and $\varphi^{\prime}(0)=B$ (indeed, $B=[Y, A]:=Y A-A Y$ for some $Y \in \mathcal{M}_{n}[13$, proof of Proposition 3] and then the mapping $\zeta \mapsto e^{\zeta Y} A e^{-\zeta Y}$ does the job).

The aim of this paper is to study the zeros of $B \mapsto k_{\Omega_{n}}(A ; B)$ in the remaining case, where $A$ is a derogatory matrix, and to relate it to the existence of entire curves tangent to $B$ at the point $A$ (which is an obvious sufficient condition for $\left.k_{\Omega_{n}}(A ; B)=0\right)$.

For $A \in \Omega_{n}$ denote by $C_{A}$ the tangent cone (cf. [6, p. 79] for this notion) to the isospectral variety

$$
L_{A}:=\left\{C \in \Omega_{n}: \operatorname{sp}(C)=\operatorname{sp}(A)\right\},
$$

that is,

$$
C_{A}:=\left\{B \in \mathcal{M}_{n}: \exists 0<c_{j} \rightarrow 0, C_{j} \in L_{A} \text { with } c_{j}\left(C_{j}-A\right) \rightarrow B\right\} .
$$

Observe that $L_{A}$ is smooth at $A$ if $A \in \mathcal{C}_{n}$; then $C_{A}=\operatorname{ker} \sigma_{*, A}$. When $A \notin \mathcal{C}_{n}$, the rank of $\sigma_{*, A}$ is not maximal, so we have $\operatorname{dim} \operatorname{ker} \sigma_{*, A}>n^{2}-n$; by [6, Corollary, p. 83], $C_{A}$ is an analytic set with $\operatorname{dim} C_{A}=\operatorname{dim} L_{A}=n^{2}-n$, so we have $C_{A} \subsetneq \operatorname{ker} \sigma_{*, A}$.

The following proposition characterizes the tangent cone $C_{A}$ as the set of "generalized" tangent vectors at $A$ to an entire curve in $\Omega_{n}$ through $A$ (therefore contained in $L_{A}$ ).

Proposition 1. Let $A \in \Omega_{n}$ and $B \in \mathcal{M}_{n}$. Then there are $m \in \mathbb{N}=$ $\{m \in \mathbb{Z}: m>0\}, m \leq n !$, and $\varphi \in \mathcal{O}\left(\mathbb{C}, \Omega_{n}\right)$ with $\varphi(0)=A, \varphi^{\prime}(0)=\cdots=$ $\varphi^{(m-1)}(0)=0, \varphi^{(m)}(0)=B$ if and only if $B \in C_{A}$.

Proposition 1 implies that $C_{A}$ is contained in the zero set of the singular Kobayashi pseudometric (cf. [14])

$$
\begin{aligned}
\widehat{k}_{\Omega_{n}}(A ; B)=\inf \{|\alpha|: \exists m \in \mathbb{N}, \varphi & \in \mathcal{O}\left(\mathbb{D}, \Omega_{n}\right): \\
& \left.\operatorname{ord}_{0}(\varphi-z) \geq m, \alpha \varphi^{(m)}(0)=m ! X\right\} .
\end{aligned}
$$

A consequence of the proof of Proposition 1 is the following.

Corollary 2. Let $A \in \Omega_{n}$ and $B \in C_{A}$. Then the following conditions are equivalent:

(a) There is $\varphi \in \mathcal{O}\left(\mathbb{C}, \Omega_{n}\right)$ with $\varphi(0)=A$ and $\varphi^{\prime}(0)=B$. 
(b) There are $r_{j} \rightarrow \infty$ and $\varphi_{j} \in \mathcal{O}\left(r_{j} \mathbb{D}, \Omega_{n}\right), j \in \mathbb{N}$, uniformly bounded near 0 , such that $\varphi_{j}(0)=A$ and $\varphi_{j}^{\prime}(0)=B$.

(c) There are $r>0$ and $\varphi \in \mathcal{O}\left(r \mathbb{D}, L_{A}\right)$ with $\varphi(0)=A$ and $\varphi^{\prime}(0)=B$.

Before stating the next proposition, we shall define an algebraic cone $C_{A}^{\prime} \subset \mathcal{M}_{n}, A \in \Omega_{n}$.

For a function $g$ holomorphic near $A$, and $X$ in a neighborhood of $A$, let $g(X)-g(A)=g_{A}^{*}(X-A)+\cdots$, where $g_{A}^{*}$ stands for the homogeneous polynomial of lowest nonzero degree in the expansion of $g$ near $A$ (the omitted terms are thus of higher order).

Set

$$
\begin{aligned}
& C_{A}^{*}:=\left\{B \in \mathcal{M}_{n}:\left(\sigma_{1}\right)_{A}^{*}(B)=0, \ldots,\left(\sigma_{n}\right)_{A}^{*}(B)=0\right\}, \\
& C_{A}^{\prime}:=\bigcap_{\lambda \in \operatorname{sp}(A)}\left(\Phi_{\lambda}\right)_{*, A}^{-1}\left(C_{\Phi_{\lambda}(A)}^{*}\right),
\end{aligned}
$$

where

$$
\Phi_{\lambda}(A):=(A-\lambda I)(I-\bar{\lambda} A)^{-1} .
$$

Note that

$$
C_{A} \subset C_{A}^{*} \subset \operatorname{ker} \sigma_{*, A} .
$$

For the first inclusion, see [6, p. 86, lines 4-6]), and for the second one, use the fact that

$$
\operatorname{ker} \sigma_{*, A}=\left\{\left(\sigma_{j}\right)_{A}^{*}=0 \text { for all } j \text { such that } \operatorname{deg}\left(\sigma_{j}\right)_{A}^{*}=1\right\} .
$$

Since $C_{A}$ and ker $\sigma_{*, A}$ are invariant under automorphisms of $\Omega_{n}$, it follows that

$$
C_{A} \subset C_{A}^{\prime} \subset \operatorname{ker} \sigma_{*, A}
$$

Moreover, if $\operatorname{dim} C_{A}=\operatorname{dim} C_{A}^{*}$, that is, $\operatorname{dim} C_{A}^{*}=n^{2}-n$, then $C_{A}=$ $C_{A}^{*}=C_{A}^{\prime}$ (cf. [6, p. 112, Corollary 2]).

Proposition 3. Let $A \in \Omega_{n} \backslash \mathcal{C}_{n}$.

(i) If $\widehat{k}_{\Omega_{n}}(A ; B)=0$, then $B \in C_{A}^{\prime}$.

(ii) $C_{A}^{\prime} \neq \operatorname{ker} \sigma_{*, A}$.

REMARK. The cone $C_{A}^{*}$ may coincide with ker $\sigma_{*, A}$ for some $A \in \Omega_{n} \backslash \mathcal{C}_{n}$, $n \geq 3$. For example, if $A:=\operatorname{diag}(t, \ldots, t, 0), t \in \mathbb{D}_{*}$, then

$$
C_{A}^{*}=\operatorname{ker} \sigma_{*, A}=\left\{B \in \mathcal{M}_{n}: \operatorname{tr} B=b_{n n}=0\right\} .
$$

The main consequence of Proposition 3 is that for $A \in \Omega_{n} \backslash \mathcal{C}_{n}$ and $B \in$ $\operatorname{ker} \sigma_{*, A} \backslash C_{A}^{\prime}$, a lifting for the corresponding Carathéodory-Fejér problem is not possible and $k_{\Omega_{n}}(\cdot ; B)$ is not a continuous function at $A$. This generalizes previous discontinuity results (see [13] and references therein).

Note also that the cone $C_{A}^{\prime}$ may coincide with $C_{A}$ in some cases, for example, for any $A \in \Omega_{2}$ (then also $C_{A}^{*}=C_{A}$ ) and any $A \in \Omega_{3}$ (see 
Proposition 6 and the discussion before it). We do not know whether this holds in general. On the other hand, it is not hard to find cases where $C_{A} \subsetneq C_{A}^{*}$.

Proposition 4. For any $n \geq 3$ there is $A \in \Omega_{n}$ such that $C_{A} \subsetneq C_{A}^{*}$.

Now, we state a conjecture about the zero set of $k_{\Omega_{n}}$.

Conjecture $5 . k_{\Omega_{n}}(A ; B)=0$ if and only if there is $\varphi \in \mathcal{O}\left(\mathbb{C}, \Omega_{n}\right)$ with $\varphi(0)=A$ and $\varphi^{\prime}(0)=B$. In particular, if $k_{\Omega_{n}}(A ; B)=0$, then $B \in C_{A}$.

Conversely, however, there are matrices $B \in C_{A}$ such that $k_{\Omega_{n}}(A ; B) \neq 0$ (see Proposition 6(ii) and Corollary 7).

There are some cases where our conjecture can be checked.

For example, since $\Omega_{n}$ is a balanced domain, $l_{\Omega_{n}}(0, \cdot)$ and $k_{\Omega_{n}}(0 ; \cdot)$ coincide with the Minkowski function, that is, with the spectral radius. Thus the zeros of $k_{\Omega_{n}}(0 ; \cdot)$ are exactly the zero-spectrum matrices, and the set of those matrices is a union of complex lines through 0 .

Also, if $A$ is a scalar matrix, that is, $A=\lambda I, \lambda \in \mathbb{C}$, then $B \in C_{A}$ if and only if there is $\varphi \in \mathcal{O}\left(\mathbb{C}, \Omega_{n}\right)$ with $\varphi(0)=A$ and $\varphi^{\prime}(0)=B$. To see this, use an automorphism of $\Omega_{n}$ of the form (1) to reduce to the case $A=0$.

Since the derogatory matrices in $\Omega_{2}$ are exactly the scalar matrices, we may choose $m=1$ in Proposition 1 if $n=2$, and $C_{A}$ coincides both with the zeros of $k_{\Omega_{2}}(A ; \cdot)$ and with the matrices $B=\varphi^{\prime}(0)$ for some entire curve $\varphi$ in $\Omega_{2}$ (on the other hand, $\operatorname{ker} \sigma_{*, A}=\left\{B \in \mathcal{M}_{2}: \operatorname{tr} B=0\right\}$ ).

Now we shall study the zero set of $k_{\Omega_{3}}(A ; \cdot)$, when $A$ is a nonscalar derogatory matrix. Using first an automorphism of the form (1) and then an automorphism of the form $C \mapsto D^{-1} C D$ reduces the problem to the following two cases:

$$
A=A_{t}:=\left(\begin{array}{ccc}
0 & 0 & 0 \\
0 & 0 & 0 \\
0 & 0 & t
\end{array}\right), \quad t \in \mathbb{D}_{*}, \quad A=\widetilde{A}:=\left(\begin{array}{ccc}
0 & 0 & 0 \\
0 & 0 & 1 \\
0 & 0 & 0
\end{array}\right) .
$$

It is easy to see that

$$
\begin{aligned}
C_{A_{t}} \subset C_{A_{t}}^{*}=C_{A_{t}}^{\prime} & =\left\{B \in \mathcal{M}_{3}: b_{33}=b_{11}+b_{22}=b_{11}^{2}+b_{12} b_{21}=0\right\}, \\
C_{\widetilde{A}} \subset C_{\widetilde{A}}^{\prime} & =\left\{B \in \mathcal{M}_{3}: b_{11}+b_{22}+b_{33}=b_{32}=b_{12} b_{31}=0\right\}
\end{aligned}
$$

(to prove, for example, the second inclusion, use the fact that if $B_{\varepsilon}=A+$ $\varepsilon B+o(\varepsilon)$, then $\operatorname{tr} B_{\varepsilon}=\varepsilon \operatorname{tr} B+o(\varepsilon), \sigma_{2}\left(B_{\varepsilon}\right)=-\varepsilon b_{32}+o(\varepsilon)$ and $\operatorname{det} B_{\varepsilon}=$ $\left.\varepsilon^{2}\left(b_{12} b_{31}-b_{11} b_{32}\right)+o\left(\varepsilon^{2}\right)\right)$. The next proposition implies, in particular, that $C_{A_{\lambda}}=C_{A_{\lambda}}^{\prime}$ and $C_{\widetilde{A}}=C_{\widetilde{A}}^{\prime}$ (use the fact that the tangent cones are closed or the dimensional reasoning mentioned above). 
Proposition 6.

(i) For any $B \in C_{A_{t}}^{\prime}(t \neq 0)$ there is $\varphi \in \mathcal{O}\left(\mathbb{C}, \Omega_{3}\right)$ with $\varphi(0)=A_{t}$ and $\varphi^{\prime}(0)=B$.

(ii) Let $B \in C_{\widetilde{A}}^{\prime}$. Then there is $\varphi \in \mathcal{O}\left(\mathbb{C}, \Omega_{n}\right)$ with $\varphi(0)=\widetilde{A}$ and $\varphi^{\prime}(0)=$ $B$ if and only if $b_{11}=0$ or $b_{12} \neq b_{31}$. Otherwise, $k_{\Omega_{3}}(\widetilde{A} ; B)>0$.

Corollary 7. For any $n \geq 3$ there are $A \in \Omega_{n}$ and $B \in C_{A}$ such that $k_{\Omega_{n}}(A ; B)>0$.

Since $k_{\Omega_{3}}(A ; B)>0$ if $B \notin C_{A}^{\prime}$, Proposition 6 and the discussion before give a complete description of the zero set of $k_{\Omega_{3}}$.

Note that the situation is much easier for the Carathéodory-Reiffen pseudometric

$$
\gamma_{\Omega_{n}}(A ; B)=\sup \left\{\left|f^{\prime}(A) B\right|: f \in \mathcal{O}(D, \mathbb{D})\right\} .
$$

Here $\gamma_{\Omega_{n}}(A ; B)=0$ if and only if $\sigma_{*, A}(B)=0$. Indeed, if $\sigma_{*, A}(B) \neq 0$, then

$$
\gamma_{\Omega_{n}}(A ; B) \geq \gamma_{\mathbb{G}_{n}}\left(A ; \sigma_{*, A}(B)\right)>0 .
$$

On the other hand, if $A \in \mathcal{C}_{n}$ and $\sigma_{*, A}(B)=0$, then

$$
0=k_{\Omega_{n}}(A ; B) \geq \gamma_{\Omega_{n}}(A ; B) \geq 0 .
$$

It remains to use the density of $\mathcal{C}_{n}$ in $\Omega_{n}$ and the continuity of the Carathéodory-Reiffen pseudometric.

The rest of the paper is organized as follows. The proofs of Propositions 3 and 4 are given in Section 2, the proofs of Proposition 6 and Corollary 7 in Section 3, and the proofs of Proposition 1 and Corollary 2 in Section 4.

Acknowledgments. The authors wish to thank the referee for a quick, thorough and perceptive report, thanks to which several mistakes in the first version have been corrected.

\section{Proofs of Propositions 3 and 4}

Proof of Proposition 4. Set $A:=\operatorname{diag}(0, \ldots, 0, t, t), t \in \mathbb{D}_{*}$. It is easy to see that

$$
\begin{aligned}
& \left(\sigma_{1}\right)_{A}^{*}(B)=\sum_{j=1}^{n} b_{j j} \\
& \left(\sigma_{2}\right)_{A}^{*}(B)=2 t \sum_{j=1}^{n-2} b_{j j}+t\left(b_{n-1, n-1}+b_{n n}\right), \\
& \left(\sigma_{3}\right)_{A}^{*}(B)=t^{2} \sum_{j=1}^{n-2} b_{j j} .
\end{aligned}
$$

Therefore, $\left(\sigma_{3}\right)_{A}^{*}=t\left(\sigma_{2}\right)_{A}^{*}-t^{2}\left(\sigma_{1}\right)_{A}^{*}$ and $\operatorname{dim} C_{A}^{*}>n^{2}-n=\operatorname{dim} C_{A}$. 
Proof of Proposition 3. (i) Let $\widehat{\gamma}_{\Omega_{n}}(A ; B)$ be the singular Carathéodory metric (cf. [12])

$$
\widehat{\gamma}_{\Omega_{n}}(A ; B):=\sup \left\{\left|\frac{f^{(k)}(A) B}{k !}\right|^{1 / k}: k \in \mathbb{N}, f \in \mathcal{O}\left(\Omega_{n}, \mathbb{D}\right), \operatorname{ord}_{A} f \geq k\right\},
$$

where $\left|\frac{f^{(k)}(A) B}{k !}\right|=\sum_{|\alpha|=k} D^{\alpha} f(A) B^{\alpha}$. Since

$$
\widehat{k}_{\Omega_{n}}(A ; B) \geq \widehat{\gamma}_{\Omega_{n}}(A ; B),
$$

it is enough to show that $\widehat{\gamma}_{\Omega_{n}}(A ; B)>0$ if $B \notin C_{A}^{\prime}$. Then $B \in C_{\Phi_{\lambda}(A)}^{*}$ for some $\lambda \in \operatorname{sp}(A)$. Replacing $A$ and $B$ by $\Phi_{\lambda}(A)$ and $\left(\Phi_{\lambda}\right)_{*, A}(B)$, respectively, we may assume that $B \notin C_{A}^{*}$. Then there is $\sigma_{j}$ such that $\left(\sigma_{j}\right)_{A}^{*}(B) \neq 0$. Denoting by $k$ the degree of $\left(\sigma_{j}\right)_{A}^{*}$, it follows that

$$
\widehat{\gamma}_{\Omega_{n}}(A ; B) \geq\left|\frac{\left(\sigma_{j}\right)_{A}^{*}(B)}{\left(\begin{array}{c}
n \\
j
\end{array}\right)}\right|^{1 / k}>0 .
$$

(ii) Since $A \in \Omega_{n} \backslash \mathcal{C}_{n}$, at least two of the eigenvalues of $A$ are equal, say to $\lambda$. Applying the automorphism $\Phi_{\lambda}$ of $\Omega_{n}$, we may assume that $\lambda=0$. Since the map $A \mapsto P^{-1} A P$ is a linear automorphism of $\Omega_{n}$ for any $P \in \mathcal{M}_{n}^{-1}$, we may also assume that $A$ is in Jordan form. In particular,

$$
A=\left(\begin{array}{cc}
A_{0} & 0 \\
0 & A_{1}
\end{array}\right)
$$

where $A_{0} \in \mathcal{M}_{m}, 2 \leq m \leq n, \operatorname{sp}\left(A_{0}\right)=\{0\}, A_{1} \in \mathcal{M}_{n-m}, 0 \notin \operatorname{sp}\left(A_{1}\right)$. Furthermore, there is a set $J \subsetneq\{2, \ldots, m\}$, possibly empty, such that $a_{j-1, j}=1$ for $j \in J$, and all other coefficients $a_{i j}$ are zero for $1 \leq i, j \leq m$. Define $0 \leq r:=\# J=\operatorname{rank} A_{0} \leq m-2$.

We set

$$
B:=\left(\begin{array}{cc}
B_{0} & 0 \\
0 & 0
\end{array}\right) \in \mathcal{M}_{n}
$$

where $B_{0}=\left(b_{i j}\right)_{1 \leq i, j \leq m}$ is such that $b_{j-1, j}=-1$ for $j \in\{2, \ldots, m\} \backslash J$, $b_{m 1}=1$, and $b_{i j}=0$ otherwise. To complete the proof, it is enough to show the following.

Lemma 8. $\left(\sigma_{m}\right)_{A}^{*}(B)=1$, but $\sigma_{*, A}(B)=0$.

Proof. We begin by computing $\sigma_{j}\left(A_{0}+h B_{0}\right), 1 \leq j \leq m, h \in \mathbb{C}$. Expanding with respect to the first column, we see that

$$
\operatorname{det}\left(t I-\left(A_{0}+h B_{0}\right)\right)=t^{m}+(-1)^{m-1} h^{m-r} .
$$

Comparing the corresponding coefficients of both sides, it follows that

$$
\sigma_{j}\left(A_{0}+h B_{0}\right)= \begin{cases}0, & 1 \leq j \leq m-1 \\ h^{m-r}, & j=m\end{cases}
$$


Next, we need a general formula for the functions $\sigma_{j}$. Given a matrix $M=$ $\left(m_{i j}\right)_{1 \leq i, j \leq n}$ and a set $E \subset\{1, \ldots, n\}$, we write $\delta_{E}(M)$ for the determinant of the matrix $\left(m_{i j}\right)_{i, j \in E} \in \mathcal{M}_{\# E}$. By convention, $\delta_{\emptyset}(M)=\sigma_{0}(M):=1$. Then

$$
\sigma_{j}(M)=\sum_{E \subset\{1, \ldots, n\}, \# E=j} \delta_{E}(M)
$$

Because of the block structure of our matrices,

$$
\delta_{E}(A+h B)=\delta_{E \cap\{1, \ldots, m\}}\left(A_{0}+h B_{0}\right) \delta_{E \cap\{m+1, \ldots, n\}}\left(A_{1}\right) .
$$

Therefore

$$
\begin{aligned}
\sigma_{j}(A+h B)= & \sum_{\max (0, j-n+m) \leq k \leq \min (m, j)}\left(\sum_{E^{\prime} \subset\{1, \ldots, m\}, \# E^{\prime}=k} \delta_{E^{\prime}}\left(A_{0}+h B_{0}\right)\right) \\
& \times\left(\sum_{E^{\prime \prime} \subset\{m+1, \ldots, n\}, \# E^{\prime \prime}=j-k} \delta_{E^{\prime \prime}}\left(A_{1}\right)\right) \\
= & \sum_{\max (0, j-n+m) \leq k \leq \min (m, j)} \sigma_{k}\left(A_{0}+h B_{0}\right) \sigma_{j-k}\left(A_{1}\right) .
\end{aligned}
$$

It follows by (2) that $\sigma_{j}(A+h B)=S_{1}+S_{2}$, where

$$
S_{1}=\left\{\begin{array}{ll}
\sigma_{j}\left(A_{1}\right), & j \leq n-m, \\
0, & \text { otherwise },
\end{array} \quad S_{2}= \begin{cases}h^{m-r} \sigma_{j-m}\left(A_{1}\right), & j \geq m, \\
0, & \text { otherwise }\end{cases}\right.
$$

In particular,

$$
\sigma_{j}(A)= \begin{cases}\sigma_{j}\left(A_{1}\right), & j \leq n-m \\ 0, & \text { otherwise }\end{cases}
$$

Then

$$
\sigma_{j}(A+h B)-\sigma_{j}(A)= \begin{cases}h^{m-r} \sigma_{j-m}\left(A_{1}\right), & j \geq m, \\ 0, & \text { otherwise. }\end{cases}
$$

Since $m-r \geq 2$ we conclude that $\sigma_{*, A}(B)=0$, but $\left(\sigma_{m}\right)_{A}^{*}(B)=1$.

\section{Proofs of Proposition 6 and Corollary 7}

Proof of Proposition 6. (i) Let first $B \in C_{A_{t}}^{\prime}$. We shall write $B$ in the form $B=X+\left[Y, A_{t}\right]$, where $X$ is such that $\psi(\zeta)=A_{t}+\zeta X \in L_{A_{t}}$ for any $\zeta \in \mathbb{C}$. Then $\varphi(\zeta)=e^{\zeta Y} \psi(\zeta) e^{-\zeta Y}$ has the required properties.

It is easy to compute that $\psi(\mathbb{C}) \subset L_{A_{t}}$ if and only if $\operatorname{sp}(X)=\{0\}$ and $x_{11}+x_{22}=x_{11}^{2}+x_{12} x_{21}=0$. On the other hand,

$$
\left[Y, A_{t}\right]=t\left(\begin{array}{ccc}
0 & 0 & y_{13} \\
0 & 0 & y_{23} \\
-y_{31} & -y_{32} & 0
\end{array}\right)
$$


So we may take

$$
X=\left(\begin{array}{ccc}
b_{11} & b_{12} & 0 \\
b_{21} & b_{22} & 0 \\
0 & 0 & 0
\end{array}\right), \quad Y=t^{-1}\left(\begin{array}{ccc}
0 & 0 & b_{13} \\
0 & 0 & b_{23} \\
-b_{31} & -b_{32} & 0
\end{array}\right) .
$$

(ii) Let first $B \in \mathbb{C}_{\widetilde{A}}^{\prime}$. If $b_{11}=0$ or $b_{12} \neq b_{31}$, it is enough to find (as above) $X$ and $Y$ such that $B=X+[Y, \widetilde{A}]$ and $\widetilde{A}+\zeta X \in L_{\widetilde{A}}$ for any $\zeta \in \mathbb{C}$. The last condition means that $\operatorname{sp}(X)=\{0\}$ and $x_{32}=x_{12} x_{31}=0$. On the other hand,

$$
[Y, \widetilde{A}]=\left(\begin{array}{ccc}
0 & 0 & y_{12} \\
-y_{31} & -y_{33} & y_{22}-y_{33} \\
0 & 0 & y_{32}
\end{array}\right)
$$

Assume that $b_{31}=0$ (the computations are similar in the case $b_{12}=0$ ). Then we have to choose $X$ of the form

$$
X=\left(\begin{array}{ccc}
b_{11} & b_{12} & b_{13}-y_{12} \\
b_{21}+y_{31} & b_{22}+y_{32} & b_{23}-y_{22}+y_{33} \\
0 & 0 & -b_{11}-b_{22}-y_{32}
\end{array}\right)
$$

such that $\operatorname{det} X=0$ and $\sigma_{2}(X)=0$, that is, $D T=0, D=T^{2}$, where we write

$$
D:=\left|\begin{array}{cc}
b_{11} & b_{12} \\
b_{21}+y_{31} & b_{22}+y_{32}
\end{array}\right|, \quad T:=b_{11}+b_{22}+y_{32} .
$$

These conditions are satisfied if and only if

$$
y_{32}=-b_{11}-b_{22}, \quad y_{31}= \begin{cases}-b_{21}, & b_{11}=0 \\ -b_{21}-b_{11}^{2} / b_{12}, & b_{12} \neq 0 .\end{cases}
$$

It remains to show that if $b_{11} \neq 0$ and $b_{12}=b_{31}=0$, then $k_{\Omega_{3}}(\widetilde{A} ; B)>0$. We may assume that $b_{11}=1$. Set

$$
\widetilde{X}=\left(\begin{array}{ccc}
1 & 0 & 0 \\
0 & -1 & 0 \\
0 & 0 & 0
\end{array}\right)
$$

Choosing $X$ and $Y$ as above yields $B=\widetilde{X}+\left[Y, A_{t}\right]$. Let $\alpha>0$ and $\varphi \in \mathcal{O}\left(\alpha \mathbb{D}, \Omega_{3}\right)$ be such that $\varphi(0)=A_{t}$ and $\varphi^{\prime}(0)=B$. Setting $\widetilde{\varphi}(\zeta)=$ $e^{-\zeta Y} \varphi(\zeta) e^{\zeta Y}$, we have $\widetilde{\varphi} \in \mathcal{O}\left(\alpha \mathbb{D}, \Omega_{3}\right), \widetilde{\varphi}(0)=\widetilde{A}$ and $\widetilde{\varphi}^{\prime}(0)=X$. It follows that $k_{\Omega_{3}}\left(A_{t} ; B\right) \geq k_{\Omega_{3}}\left(A_{t} ; X\right)$. The opposite inequality follows in the same way. 
Write $\widetilde{\varphi}$ in the form

$$
\widetilde{\varphi}(\zeta)=\widetilde{A}+\zeta \widetilde{X}+\zeta^{2} \widehat{X}+o\left(\zeta^{2}\right) .
$$

Then we compute that

$$
\sigma_{2}(\widetilde{\varphi}(\zeta))=\zeta^{2}\left(1-\widehat{x}_{32}\right)+o\left(\zeta^{2}\right), \quad \operatorname{det} \widetilde{\varphi}(\zeta)=-\zeta^{3} \widehat{x}_{32}+o\left(\zeta^{3}\right) .
$$

Since $\left|\sigma_{2} \circ \varphi\right|<3$ and $|\operatorname{det} \varphi|<1$, by the Cauchy inequalities we get

$$
\left|\widehat{x}_{32}-1\right| \leq 3 \alpha^{-2}, \quad\left|\widehat{x}_{32}\right| \leq \alpha^{-3} \text {. }
$$

So

$$
k_{\Omega_{3}}\left(A_{t} ; B\right)=k_{\Omega_{3}}(\tilde{A} ; \tilde{X}) \geq \min _{t \in \mathbb{C}} \max \{\sqrt{|t-1| / 3}, \sqrt[3]{|t|}\}>0 .
$$

Proof of Corollary 7. Set

$$
\begin{array}{ll}
\widetilde{A}=\left(\begin{array}{ccc}
0 & 0 & 0 \\
0 & 0 & 1 \\
0 & 0 & 0
\end{array}\right), & \widetilde{B}_{\varepsilon}=\left(\begin{array}{ccc}
1 & \varepsilon & 0 \\
0 & -1 & 0 \\
0 & 0 & 0
\end{array}\right), \\
A=\left(\begin{array}{cc}
\widetilde{A} & O \\
O & O
\end{array}\right), & B_{\varepsilon}=\left(\begin{array}{cc}
\widetilde{B}_{\varepsilon} & O \\
O & O
\end{array}\right) .
\end{array}
$$

It follows as in the proof of Proposition 6(ii) that

- $k_{\Omega_{n}}\left(A ; B_{0}\right)>0$;

- for $\varepsilon \neq 0$, there is $\varphi_{\varepsilon} \in \mathcal{O}\left(\mathbb{C}, \Omega_{n}\right)$ with $\varphi_{\varepsilon}(0)=A$ and $\varphi_{\varepsilon}^{\prime}(0)=B_{\varepsilon}$.

Then $B_{\varepsilon} \in C_{A}$ for $\varepsilon \neq 0$, and hence $B_{0} \in C_{A}$.

\section{Proofs of Proposition 1 and Corollary 2}

Proof of Corollary 2. The implication $(\mathrm{a}) \Rightarrow(\mathrm{b})$ is trivial and the main implication $(\mathrm{c}) \Rightarrow(\mathrm{a})$ is a particular case of Proposition 9 below.

It remains to prove that $(\mathrm{b}) \Rightarrow(\mathrm{c})$. Let $\psi(\zeta)=\sum_{k=0}^{\infty} A_{k} \zeta^{k} \in \mathcal{O}\left(s \mathbb{D}, \Omega_{n}\right)$ for some $s>0$. Let $a_{k, \psi} \in \mathbb{C}^{(k+1) \times n^{2}}$ be the vector with components the entries of $A_{0}, \ldots, A_{k}$ (taken in some order). Note that

$$
\sigma_{l}(\psi(\zeta))=\sum_{k=0}^{\infty} p_{l, k}\left(a_{k, \psi}\right) \zeta^{k}, \quad 1 \leq l \leq n,
$$

where the $p_{l, k}$ are polynomials.

Let now $r_{j} \rightarrow \infty$ and $\varphi_{j} \in \mathcal{O}\left(r_{j} \mathbb{D}, \Omega_{n}\right), j \in \mathbb{N}$, uniformly bounded near 0 , be such that $\varphi_{j}(0)=A$ and $\varphi_{j}^{\prime}(0)=B$. Then we may assume that $\varphi_{j} \rightarrow \varphi \in \mathcal{O}\left(r \mathbb{D}, \Omega_{n}\right)$ for some $r>0$. Hence $p_{l, k}\left(a_{k, \varphi_{j}}\right) \rightarrow p_{l, k}\left(a_{k, \varphi}\right)$. On the other hand, $\left|p_{l, k}\left(a_{k, \varphi_{j}}\right)\right| \leq\left(\begin{array}{l}n \\ l\end{array}\right) / r_{j}^{k} \rightarrow 0, k>0$, by the Cauchy inequalities. Hence $p_{l, k}\left(a_{k, \varphi}\right)=0, k>0$, that is, $\sigma_{l}(\varphi(\zeta))=\sigma_{l}(\varphi(0))=\sigma_{l}(A)$. This means that $\varphi(\zeta) \in L_{A}$. 
Proof of Proposition 1. It is clear that if such a $\varphi$ exists, then $B \in C_{A}$.

Conversely, let $B \in C_{A}$. Then, by [6, p. 86, Proposition 1], there exists a one-dimensional irreducible analytic variety $L_{A, B} \subset L_{A}$, tangent to $B$ at $A$. Now, by $[6$, p. 80 , Proposition], there exist $m \in \mathbb{N}, r>0$ and $\psi \in$ $\mathcal{O}\left(r \mathbb{D}, L_{A, B}\right)$ such that $\psi(\zeta)=A+\zeta^{m} B+o\left(\zeta^{m}\right)$.

The integer $m$ is the number of sheets in the (local) branched covering provided by the orthogonal projection from $L_{A}$ to a suitable linear subspace of dimension $n^{2}-n$ (see [6]). This number of sheets corresponds to the cardinality of the solution set, in each generic fiber of the projection, of the equations $\sigma_{j}(M)=\sigma_{j}(A), 1 \leq j \leq n$. Bézout's theorem shows that this cardinality is less than or equal to the product of the degrees of the polynomials, so here $m \leq n$ !.

The above considerations will prove the estimate in Proposition 1, provided that we can replace $\psi$ by an entire map $\widetilde{\psi}$ with the same expansion up to order $m$ near 0 . So the proof of Proposition 1 reduces to the following.

Proposition 9. If $A \in \Omega_{n}, m \in \mathbb{N}$ and $\psi \in \mathcal{O}\left(r \mathbb{D}, L_{A}\right)$ for some $r>0$, then there is $\widetilde{\psi} \in \mathcal{O}\left(\mathbb{C}, L_{A}\right)$ with $\widetilde{\psi}(\zeta)=\psi(\zeta)+o\left(\zeta^{m}\right)$.

Proof. We want to reduce the problem by replacing each matrix $\psi(\zeta)$ by a conjugate matrix $\varphi(\zeta)$ (in particular, they will have the same spectrum, so we remain inside $L_{A}$ and inside $\Omega_{n}$ ). If we can manage this so that $\varphi(\zeta)$ is upper triangular, then an entire map with the same spectrum matching $\varphi$ up to order $m$ can be obtained by taking the Taylor polynomial of degree $m$ of each coefficient of $\varphi$.

To proceed with this program, first we need to show that conjugation (with a holomorphic change of basis) does not change the problem.

Let $\mathcal{M}_{n}^{-1}$ stand for the group of all invertible $n \times n$ matrices.

Lemma 10. Let $r>0, P \in \mathcal{O}\left(r \mathbb{D}, \mathcal{M}_{n}^{-1}\right)$ and $\psi \in \mathcal{O}\left(r \mathbb{D}, \Omega_{n}\right)$. Write $\varphi(\zeta):=P(\zeta)^{-1} \psi(\zeta) P(\zeta)$, and assume that there exists $\widetilde{\varphi} \in \mathcal{O}\left(\mathbb{C}, \Omega_{n}\right)$ such that near $0, \widetilde{\varphi}(\zeta)=\varphi(\zeta)+o\left(\zeta^{m}\right)$. Then there exists $\widetilde{\psi} \in \mathcal{O}\left(\mathbb{C}, \Omega_{n}\right)$ conjugate to $\widetilde{\varphi}$ (in particular having the same spectrum) such that near 0 ,

$$
\widetilde{\psi}(\zeta)=\psi(\zeta)+o\left(\zeta^{m}\right)
$$

Note once again that Liouville's theorem implies that the entire maps $\widetilde{\varphi}, \widetilde{\psi}$ actually map to $L_{\widetilde{\varphi}(0)}=L_{\widetilde{\psi}(0)}$.

Proof. Note first that, because the exponential is locally Lipschitz, $\exp (A+M)=\exp A+O(M)$.

Denote by $L_{m}(x)$ the Taylor polynomial of degree $m$ at 0 for the function $x \mapsto \ln (1+x)$. Since $\exp (\ln (1+x))=1+x$ and $\ln (1+x)=L_{m}(x)+o\left(x^{m}\right)$, we have $\exp \left(L_{m}(x)\right)=1+x+o\left(x^{m}\right)$. So $\exp \left(L_{m}(A)\right)=I+A+o\left(A^{m}\right)$. 
Now write

$$
P(\zeta)=P(0)\left(I+\sum_{k=1}^{m} A_{k} \zeta^{k}+O\left(\zeta^{m+1}\right)\right)=: P(0)(I+M(\zeta)) .
$$

Define $P_{1}$ to be the unique matrix-valued polynomial of degree $\leq m$ in $\zeta$ so that

$$
L_{m}\left(\sum_{k=1}^{m} A_{k} \zeta^{k}\right)=P_{1}(\zeta)+o\left(\zeta^{m}\right) .
$$

Then, remarking that $M(\zeta)=o(1)$, we have

$$
\exp \left(P_{1}(\zeta)\right)=\exp \left(L_{m}(M(\zeta))+o\left(\zeta^{m}\right)=I+M(\zeta)+o\left(\zeta^{m}\right),\right.
$$

so that $P(0) \exp \left(P_{1}(\zeta)\right)=P(\zeta)+o\left(\zeta^{m}\right)$. Then it is easy to see that $\exp \left(-P_{1}(\zeta)\right) P(0)^{-1}=P(\zeta)^{-1}+o\left(\zeta^{m}\right)$, and $\widetilde{P}(\zeta):=P(0) \exp \left(P_{1}(\zeta)\right)$ defines an entire map. So $\widetilde{\psi}:=\widetilde{P} \widetilde{\varphi} \widetilde{P}^{-1}$ satisfies the requirements.

We now reduce the proof of Proposition 9 to the case of nilpotent matrices (that is, $\operatorname{sp}(A)=\{0\}$ ).

Lemma 11. Suppose that the conclusion of Proposition 9 holds with the additional hypothesis that $\operatorname{sp}(A)=\{0\}$. Then it holds for an arbitrary matrix A.

Proof. Write $\operatorname{sp}(\psi(\zeta))=\operatorname{sp}(A)=\left\{\mu_{j}: 1 \leq j \leq k\right\}$ where the $\mu_{j}$ are distinct eigenvalues with respective algebraic multiplicities $m_{j}$. Let $S_{j}(\zeta)=$ $\operatorname{ker}\left(\psi(\zeta)-\mu_{j} I\right)^{m_{j}}$ be the associated generalized eigenspace. Choose a basis $\left\{e_{1}, \ldots, e_{n}\right\}$ of $\mathbb{C}^{n}$ such that $S_{j}(0)=\operatorname{span}\left\{e_{i}: 1 \leq i-\sum_{l=1}^{j-1} m_{l} \leq m_{j}\right\}$. By Lemma 10, without loss of generality we may assume that the matrices are written in this basis, and therefore $\psi(0)$ is a block matrix.

By continuity of the various determinants involved, there exists some $r^{\prime}>0$ such that for $|\zeta|<r^{\prime} \leq r$, we still have, for each $j, \mathbb{C}^{n}=S_{j}(\zeta) \oplus$ $\bigoplus_{l: l \neq j} S_{l}(0)$. Then there is a unique linear projection $\pi_{j, \zeta}$ defined on $\mathbb{C}^{n}$ such that $\pi_{j, \zeta}\left(\mathbb{C}^{n}\right)=S_{j}(\zeta)$ and $\operatorname{Ker} \pi_{j, \zeta}=\bigoplus_{l: l \neq j} S_{l}(0)$. This restricts to a linear isomorphism from $S_{j}(0)$ to $S_{j}(\zeta)$. Therefore the vectors $\left\{\pi_{j, \zeta}\left(e_{i}\right)\right.$ : $\left.1 \leq i-\sum_{l=1}^{j-1} m_{l} \leq m_{j}\right\}$, being obtained as solution of a Cramer system of linear equations with holomorphic coefficients, depend holomorphically on $\zeta$ in $D\left(0, r^{\prime}\right)$. Thus $\left\{\pi_{j, \zeta}\left(e_{i}\right): 1 \leq i-\sum_{l=1}^{j-1} m_{l} \leq m_{j}, 1 \leq j \leq k\right\}$ form a basis of $\mathbb{C}^{n}$ adapted to the direct sum decomposition in the $S_{j}(\zeta)$. If we write $P(\zeta)$ for the matrix of the coordinates of the vectors of this new basis expressed in the standard basis, it depends holomorphically on $\zeta$ in $D\left(0, r^{\prime}\right)$, and the new matrix $\widehat{\psi}(\zeta):=P(\zeta)^{-1} \psi(\zeta) P(\zeta)$ has the same block structure 
as $\psi(0)$ :

$$
\widehat{\psi}(\zeta)=\left(\begin{array}{cccc}
\widehat{\psi}_{1}(\zeta) & 0 & \cdots & 0 \\
0 & \widehat{\psi}_{2}(\zeta) & \cdots & 0 \\
\vdots & \vdots & \ddots & \vdots \\
0 & 0 & \cdots & \widehat{\psi}_{k}(\zeta)
\end{array}\right)
$$

where $\widehat{\psi}_{j} \in \mathcal{O}\left(r^{\prime} \mathbb{D}, \Omega_{m_{j}}\right), \operatorname{sp}\left(\widehat{\psi}_{j}(\zeta)\right)=\left\{\mu_{j}\right\}$. The map $\omega_{j}$ defined by

$$
\omega_{j}(\zeta):=\left(\mu_{j} I_{m_{j}}-\widehat{\psi}_{j}(\zeta)\right)\left(I_{m_{j}}-\bar{\mu}_{j} \widehat{\psi}_{j}(\zeta)\right)^{-1}
$$

is in $\mathcal{O}\left(r^{\prime} \mathbb{D}, \Omega_{m_{j}}\right)$, and its values are nilpotent matrices. By our hypothesis there are maps $\widetilde{\omega}_{j} \in \mathcal{O}\left(\mathbb{C}, \Omega_{m_{j}}\right)$ such that $\widetilde{\omega}_{j}(\zeta)=\omega_{j}(\zeta)+o\left(\zeta^{m}\right)$ (and therefore with nilpotent values). Define

$$
\begin{aligned}
& \widetilde{\psi}_{j}(\zeta):=\left(\mu_{j} I_{m_{j}}-\widetilde{\omega}_{j}(\zeta)\right)\left(I_{m_{j}}-\bar{\mu}_{j} \widetilde{\omega}_{j}(\zeta)\right)^{-1}, \\
& \widetilde{\psi}(\zeta):=\left(\begin{array}{cccc}
\widetilde{\psi}_{1}(\zeta) & 0 & \ldots & 0 \\
0 & \widetilde{\psi}_{2}(\zeta) & \ldots & 0 \\
\vdots & \vdots & \ddots & \vdots \\
0 & 0 & \ldots & \widetilde{\psi}_{k}(\zeta)
\end{array}\right)
\end{aligned}
$$

It is easy to see that $\widetilde{\psi} \in \mathcal{O}\left(\mathbb{C}, \Omega_{n}\right)$ and $\widetilde{\psi}(\zeta)=\widehat{\psi}(\zeta)+o\left(\zeta^{m}\right)$.

LEMMA 12. If $m \in \mathbb{N}$ and $\psi \in \mathcal{O}\left(r \mathbb{D}, L_{0}\right)$ for some $r>0$, then there are $r^{\prime} \in(0, r)$ and $P \in \mathcal{O}\left(r^{\prime} \mathbb{D}, \mathcal{M}_{n}^{-1}\right)$ such that $\varphi(\zeta):=P(\zeta)^{-1} \psi(\zeta) P(\zeta)$ is a strictly upper triangular matrix for all $\zeta \in r^{\prime} \mathbb{D}$.

Proposition 9 follows from Lemma 12.

Indeed, by Lemma 11, we can make the additional hypothesis that $\psi$ has nilpotent values, that is, $\psi \in \mathcal{O}\left(r \mathbb{D}, L_{0}\right)$. By Lemma 12 , there is, for every $\zeta$ in a neighborhood of 0 , a strictly upper triangular matrix $\varphi(\zeta)=$ $P(\zeta)^{-1} \psi(\zeta) P(\zeta)$. If we replace each of the holomorphic coefficients $\varphi_{i j}(\zeta)$, $1 \leq i<j \leq n$, by its Taylor polynomial of order $m, \widetilde{\varphi}_{i j}(\zeta):=\sum_{k=0}^{m} \varphi_{i j}^{(k)}(0) \zeta^{k} / k !$, we obtain an approximation $\widetilde{\varphi}$ up to order $m$ of our mapping which is entire, and still strictly upper triangular, therefore still with spectrum reduced to 0 . Since $P(\zeta)$ depends holomorphically on $\zeta$ in a neighborhood of 0 , we may apply Lemma 10 and obtain a matrix $\widetilde{\psi}(\zeta)$ still with spectrum reduced to 0 , approximating $\psi$ to order $m$, and entire in $\zeta$.

Proof of Lemma 12. We are working with a matrix $\psi(\zeta)$ which satisfies $\psi(\zeta)^{n}=0$ for all $\zeta \in r \mathbb{D}$. For $1 \leq k \leq n$, let $r_{k}(\zeta):=\operatorname{rank}\left(\psi(\zeta)^{k}\right)$. For a matrix $M, \operatorname{rank}(M) \leq l<n$ if and only if all the minors of size $l+1$ vanish; in our case they are holomorphic functions of $\zeta$, therefore

$$
r_{k}(\zeta)=\max _{\mathbb{D}} r_{k}=: \widetilde{r}_{k}
$$


for all $\zeta \in r \mathbb{D}$ except on a discrete set. By replacing $r$ by a smaller positive number if necessary, we may assume that $r_{k}(\zeta)=\widetilde{r}_{k}$ for all $\zeta \in r \mathbb{D}_{*}:=$ $r \mathbb{D} \backslash\{0\}$. Set

$$
n_{k}:=n-\widetilde{r}_{k}=\operatorname{dim} \operatorname{ker} \psi(\zeta)^{k}, \quad \zeta \in r \mathbb{D}_{*} .
$$

It is a classical fact from linear algebra that, for a nilpotent matrix, if $p:=$ $\min \left\{k: n_{k}=n\right\}$, then $1 \leq n_{1}<\cdots<n_{p}=n_{p+1}=\cdots=n$.

For $1 \leq k \leq p$ and $\zeta \in r \mathbb{D}_{*}$, set $V_{k}(\zeta):=\operatorname{ker} \psi(\zeta)^{k}$. Since the Grassmannian $\mathcal{G}\left(n, n_{k}\right)$ is compact, we may find a sequence $\zeta_{i} \rightarrow 0$ and vector subspaces $V_{k}(0) \in \mathcal{G}\left(n, n_{k}\right)$ such that $\lim _{i \rightarrow \infty} V_{k}\left(\zeta_{i}\right)=V_{k}(0) \subset \operatorname{ker} \psi(0)^{k}$, $1 \leq k \leq p$.

Our problem will be solved if we find $\varepsilon>0$ and holomorphic mappings $v_{j} \in \mathcal{O}\left(\varepsilon \mathbb{D}, \mathbb{C}^{n}\right)$ such that $\left\{v_{j}(\zeta): 1 \leq j \leq n_{k}\right\}$ is a basis of $V_{k}(\zeta), \zeta \in \varepsilon \mathbb{D}$, $1 \leq k \leq p$. (In particular, $\lim _{\zeta \rightarrow 0} V_{k}(\bar{\zeta})=V_{k}(0)$.)

We shall proceed by induction on $k$, and on $j$ for each fixed $k$. The value of $\varepsilon$ may be reduced at each step, but we keep the same notation.

By convention we will set $n_{0}=0$, and consider $\emptyset$ as a basis of $\{0\}$. Suppose that we have already determined $\left\{v_{j}: 1 \leq j \leq n_{k}\right\}$. Choose an $r_{k+1} \times r_{k+1}$ submatrix of $\psi^{k+1}$ whose determinant, denoted $\delta_{k+1}$, is holomorphic and does not vanish on $\varepsilon \mathbb{D}_{*}$ and eliminate the unknowns corresponding to the columns of this minor; the other unknowns are then expressed in terms of the former with coefficients which are rational in the coefficients of the matrix $\psi^{k+1}$, so that we obtain meromorphic vector-valued functions $u_{i}$ on $\varepsilon \mathbb{D}$ so that $\left\{u_{i}(\zeta): 1 \leq j \leq n_{k+1}\right\}$ is a basis of $V_{k+1}(\zeta)$ for $\zeta \in \varepsilon \mathbb{D}_{*}$. Those functions are of the form $u_{i}:=f_{i} / \delta_{k+1}$, where $f_{i} \in \mathcal{O}\left(\varepsilon \mathbb{D}, \mathbb{C}^{n}\right)$.

By linear algebra, for each fixed $\zeta$, there exists a set $I(\zeta)$ of $n_{k+1}-n_{k}$ indices $i$ so that $\left\{v_{j}(\zeta), u_{i}(\zeta): 1 \leq j \leq n_{k}, i \in I(\zeta)\right\}$ form a basis of $V_{k+1}(\zeta)$.

Using the fact that all determinants that we have to compute to determine the rank of a system of vectors are meromorphic in $\varepsilon \mathbb{D}$, and reducing $\varepsilon$ if necessary, we may choose a fixed set $I$ so that $\left\{v_{j}(\zeta), u_{i}(\zeta)\right.$ : $\left.1 \leq j \leq n_{k}, i \in I\right\}$ is a basis of $V_{k+1}(\zeta)$ for all $\zeta \in \varepsilon \mathbb{D}_{*}$. Re-index the functions $\left\{u_{i}: i \in I\right\}$ as $w_{j}, n_{k}+1 \leq j \leq n_{k+1}$. Then $\left\{v_{j}(\zeta), w_{l}(\zeta): 1 \leq j \leq\right.$ $\left.n_{k}<l \leq n_{k+1}\right\}$ is a basis of $V_{k+1}(\zeta)$ for $\zeta \in \varepsilon \mathbb{D}_{*}$.

Since $\delta_{k+1}$ vanishes at most at 0 , we can multiply each vector-valued function $w_{l}$ by $\zeta^{\alpha_{l}}$, with $\alpha_{l} \in \mathbb{Z}$ chosen such that $\zeta^{\alpha_{l}} w_{l}(\zeta)$ extends to a map $\widetilde{w}_{l} \in \mathcal{O}\left(\varepsilon \mathbb{D}, \mathbb{C}^{n}\right)$ with $\widetilde{w}_{l}(0) \neq 0$.

We need to modify the $\widetilde{w}_{l}$ to ensure that we still have a system of maximal rank at the origin (this is in the spirit of the Gram-Schmidt orthogonalization process). We proceed by induction on $j \geq n_{k}+1$. Suppose we have determined $v_{j}$ as in the statement of the lemma up to some $j_{0} \geq n_{k}$ such 
that

$$
\begin{aligned}
& \operatorname{span}\left\{v_{j}(\zeta): 1 \leq j \leq j_{0}\right\} \\
& \quad=\operatorname{span}\left\{v_{j}(\zeta), \widetilde{w}_{l}(\zeta): 1 \leq j \leq n_{k}<l \leq j_{0}\right\}, \quad \zeta \in \varepsilon \mathbb{D}_{*}, \\
& \operatorname{rank}\left\{v_{j}(\zeta): 1 \leq j \leq j_{0}\right\}=j_{0}, \quad \zeta \in \varepsilon \mathbb{D} .
\end{aligned}
$$

Let $W(\zeta):=\operatorname{span}\left\{v_{1}(\zeta), \ldots, v_{j_{0}}(\zeta), \widetilde{w}_{j_{0}+1}(\zeta)\right\}, \zeta \in \varepsilon \mathbb{D}_{*}$. Then $\operatorname{dim} W(\zeta)=$ $j_{0}+1$. Again by using the compactness of the Grassmannian, we may choose a sequence $\zeta_{i} \rightarrow 0$ such that $\lim _{i \rightarrow \infty} W\left(\zeta_{i}\right)=: W(0)$ exists. Since all the $v_{j}$ and $\widetilde{w}_{j_{0}+1}$ are continuous at 0 , we easily deduce that $\operatorname{span}\left\{v_{1}(0), \ldots, v_{j_{0}}(0)\right.$, $\left.\widetilde{w}_{j_{0}+1}(0)\right\} \subset W(0)$. Choose a vector $w$ such that $\left\{v_{1}(0), \ldots, v_{j_{0}}(0), w\right\}$ is a basis of $W(0)$.

By reducing $\varepsilon$ if necessary, we may assume that there exists a linear subspace $Y$ such that $W(\zeta)$ and $Y$ form a direct sum for any $\zeta \in \varepsilon \mathbb{D}$. Then define $v_{j_{0}+1}(\zeta)$ to be the projection of the fixed vector $w$ onto $W(\zeta)$, parallel to $Y$, for $\zeta \neq 0$; in particular $v_{j_{0}+1}(0)=w$. Since $v_{j_{0}+1}(\zeta)$ is obtained by solving a system of linear equations with unique solution, it is meromorphic in a neighborhood of 0 , but it is also clearly continuous near 0 , hence holomorphic. Since the system $\left\{v_{1}(0), \ldots, v_{j_{0}}(0), v_{j_{0}+1}(0)\right\}$ is independent, $\left\{v_{1}(\zeta), \ldots, v_{j_{0}}(\zeta), v_{j_{0}+1}(\zeta)\right\}$ is also independent for $\zeta$ small enough, and since it is contained in $W(\zeta)$ by construction, it forms a basis of that subspace. Reducing $\varepsilon$ yet again if necessary, the induction may proceed.

\section{References}

[1] J. Agler and N. J. Young, The two-point spectral Nevanlinna-Pick problem, Integral Equations Operator Theory 37 (2000), 375-385.

[2] - - - The two-by-two spectral Nevanlinna-Pick problem, Trans. Amer. Math. Soc. 356 (2004), 573-585.

[3] - - - The hyperbolic geometry of the symmetrized bidisc, J. Geom. Anal. 14 (2004), $375-403$.

[4] H. Bercovici, C. Foiaş and A. Tannenbaum, A spectral commutant lifting theorem, Trans. Amer. Math. Soc. 325 (1991), 741-763.

[5] R. Brody, Compact manifolds and hyperbolicity, ibid. 235 (1978), 213-219.

[6] E. M. Chirka, Complex Analytic Sets, Kluwer, Dordrecht, 1989.

[7] C. Costara, The $2 \times 2$ spectral Nevanlinna-Pick problem, J. London Math. Soc. 71 (2005), 684-702.

[8] -, On the spectral Nevanlinna-Pick problem, Studia Math. 170 (2005), 23-55.

[9] A. Edigarian and W. Zwonek, Geometry of the symmetrized polydisc, Arch. Math. (Basel) 84 (2005), 364-374.

[10] H.-N. Huang, S. A. M. Marcantognini and N. J. Young, The spectral CarathéodoryFejér problem, Integral Equations Operator Theory 56 (2006), 229-256.

[11] M. Jarnicki and P. Pflug, Invariant distances and metrics in complex analysisrevisited, Dissertationes Math. 430 (2005).

[12] N. Nikolov, Continuity and boundary behavior of the Carathéodory metric, Math. Notes 67 (2000), 183-191. 
[13] N. Nikolov, P. J. Thomas and W. Zwonek, Discontinuity of the Lempert function and the Kobayashi-Royden metric of the spectral ball, preprint, 2007 (arXiv:math.CV/ 0704.2470).

[14] J. Yu, Singular Kobayashi metrics and finite type conditions, Proc. Amer. Math. Soc. 123 (1995), 121-130.

Institute of Mathematics and Informatics

Laboratoire Émile Picard

Bulgarian Academy of Sciences

Acad. G. Bonchev 8

UMR CNRS 5580

1113 Sofia, Bulgaria

Université Paul Sabatier

E-mail: nik@math.bas.bg

118 Route de Narbonne

F-31062 Toulouse Cedex, France

E-mail: pthomas@cict.fr

Received 5.6.2007

and in final form 13.11.2007 